Computing and Informatics, Vol. 40, 2021, 1056-1079, doi: 10.31577/cai_2021_5_1056

\title{
LAZY REPAIRING BACKTRACKING FOR DYNAMIC CONSTRAINT SATISFACTION PROBLEMS
}

\author{
Yosra Acodad, Amine Benamrane, El Houssine Bouyakhf \\ LIMIARF, Faculty of Sciences \\ Mohammed $V$ University in Rabat, Morocco \\ e-mail: \{yosra.acodad, benamraneamine\}@gmail.com, bouyakhf@mtds.com
}

\section{Imade BENELALLAM}

INSEA, National Institute of Statistics and Applied Economic

Irfane Rabat, Morocco

e-mail: i.benelallam@insea.ac.ma

\begin{abstract}
Extended Partial Dynamic Backtracking (EPDB) is a repair algorithm based on PDB. It deals with Dynamic CSPs based on ordering heuristics and retroactive data structures, safety conditions, and nogoods which are saved during the search process. In this paper, we show that the drawback of both EPDB and PDB is the exhaustive verification of orders, saved in safety conditions and nogoods, between variables. This verification affects remarkably search time, especially since orders are often indirectly deduced. Therefore, we propose a new approach for dynamically changing environments, the Lazy Repairing Backtracking (LRB), which is a fast version of EPDB insofar as it deduces orders directly through the used ordering heuristic. We evaluate LRB on various kinds of problems, and compare it, on the one hand, with EPDB to show its effectiveness compared to this approach, and, on the other hand, with MAC-2001 in order to conclude, from what perturbation rate resolving a DCSP with an efficient approach can be more advantageous than repair.
\end{abstract}

Keywords: DCSP, LRB, extended PDB, pdeg heuristic, MAC-2001

Mathematics Subject Classification 2010: 97P50, 97R40 


\section{INTRODUCTION}

In Artificial Intelligence (AI) and operation research, a large number of problems can be modeled as Constraint Satisfaction Problem (CSP). In fact, constraints are naturally present in real problems such as allocation problems [3], scheduling problems, sensor networks problems [18, etc. The concept of CSPs indicates all these problems and consists of looking for the solutions satisfying them. To increase the efficiency of the constraint satisfaction algorithms, many techniques as filtering, improved backtracking, using efficient representations and heuristics have been developed. However, these techniques assume that the complete description of the CSP instance is known and fixed in advance. This is a strong limitation when dealing with real situations [17, where problems may evolve due to changes in the environment or their execution conditions.

The notion of dynamic CSP (DCSP) [9] has been introduced to represent such situations. A DCSP is an extension of a static CSP. It can be viewed as a sequence of CSPs, where each one differs from the previous one by addition or removal of some constraints. In fact, all other possible changes of a CSP (constraints or domain modifications, variables additions or removals) can be expressed in terms of constraints additions or removals. The present paper focuses on scenarios where after solving a DCSP, some constraints have been added or changed, then, the old solution and reasoning are required to be effectively repaired.

Most DCSPs complete algorithms are based on backtracking, constraint propagation and nogood learning. The nogoods are used by intelligent back-trackers to record information regarding dead-ends in order to avoid encountering them again. They are used in Partial-order Dynamic Backtracking (PDB) [1], which considers a complete set of assignments that are incrementally modified until all constraints are satisfied, or no solution is found. The advantage of PDB is that it allows great flexibility in the backtracking strategy which affects the flexibility of the search space exploration. Extended Partial-order Dynamic Backtracking EPDB [2] exploits this flexibility by repairing solutions using variables ordering heuristics (VOHs) to build an optimal order, and by fixing the checked constraint, contrary to PDB, till its consistency.

In this paper, we propose a fast version of EPDB, the Lazy Repairing Backtracking (LRB) approach. This algorithm aims to repair solutions using EPDB advantages while eliminating unnecessary tests of orders.

This paper is organized as follows: First, we recall DCSP concept, EPDB approach and profound degree (pdeg) [1 heuristic. Then, we present the proposed LRB algorithm. Next, we evaluate the performance of this approach, compared to EPDB and MAC-2001 4, 5] on different types of problems respectively in order to show its effectiveness and to demonstrate when repairing a DCSP is more efficient than resolving it from the scratch. Finally, we extract some conclusions and directions for further research.

This paper is organized as follows: In Section 2 we summarize some backround concept useful for the rest of the paper. Then in Section 3 we present and detail our 
new repairing approch LRB. Next in Section 4 we present some empirical experiments to defend the performance of LRB approach. Before conclude, we discuss in Section 5 experiments' results in both repairing and solving configuration.

\section{BACKGROUND}

\subsection{Dynamic Constraint Satisfaction Problem}

Many real problems are dynamic, due to the environment or problem scenario changes. These changes are caused by the dynamism of the environment nature, spurious actions, incomplete knowledge, etc. A DCSP is a CSP which is adapted to changes. A problem change can be a restriction, which is an addition of constraints, or relaxation, that is an elimination of some existing constraints.

Definition 1 (CSP). A Constraint Satisfaction Problem (CSP) is a tuple $(X, D$, $C)$, where:

- $X$ is a set containing $n$ variables $\left\{x_{1}, x_{2}, \ldots, x_{n}\right\}$.

- $D$ is a set of domains $\left\{D\left(x_{1}\right), D\left(x_{2}\right), \ldots, D\left(x_{n}\right)\right\}$ for these variables, such as each $D\left(x_{i}\right)$ contains the possible values which $x_{i}$ may take.

- $C$ is a set of $m$ constraints $\left\{c_{1}, c_{2}, \ldots, c_{m}\right\}$ between variables in subsets of $X$. Each $c_{i} \in C$ expresses a relation defining which combinations of variables assignments are allowed for the variables $\operatorname{vars}\left(c_{i}\right)$ in the scope of the constraint.

Definition 2 (DCSP). Consider $P$ a dynamic constraint satisfaction problem (DCSP) 9]. $P$ is a sequence of static CSPs $P_{0}, \ldots, P_{\alpha}, P_{\alpha+1}, \ldots$, where each $P_{i}$ differs from the previous $P_{i-1}$ by the addition or removal of some constraints (we assume that all CSPs possible changes can be expressed in terms of constraint additions or removals).

If $P_{\alpha}=\left(X, D, C_{\alpha}\right)$, then $P_{\alpha+1}=\left(X, D, C_{\alpha+1}\right)$, where $C_{\alpha+1}=C_{\alpha} \pm C$, such as $C$ is a set of constraints.

\subsection{Extended Partial-Order Dynamic Backtracking}

The dynamic scheduling offers the possibility to rebuild a new order among variables during the search. The Partial-order Dynamic Backtracking (PDB) [1] is one of the approaches using this technique. However, it suffers from a lack of flexibility in backtracking strategy although using a partial order which supports dynamism.

In fact, PDB replaces the fixed order of variables, used in several approaches to solve CSPs, with a partial order that is dynamic and partially modified during the search process. It uses the concept of nogoods introduced in the Dynamic Backtracking algorithm (DBT) [10]. 
Definition 3 (Nogood). A nogood [11] is an expression of the form:

$$
\left(X_{1}=v_{1}\right) \wedge \cdots \wedge\left(X_{k}=v_{k}\right) \Rightarrow X \neq v .
$$

It is a failure explanation which can be used to represent a constraint as an implication. It is logically equivalent to the constraint:

$$
\neg\left[\left(X_{1}=v_{1}\right) \wedge \cdots \wedge\left(X_{k}=v_{k}\right) \wedge(X=v)\right]
$$

that represents a set of assignments that cannot be extended to a solution of the problem.

The order in PDB is manipulated when a nogood is generated, in order to justify an elimination when a constraint is violated, or when a failure of instantiating a variable is met due to a domain wipe out. Contrary to DBT, when a nogood is induced during the search, PDB verifies the current order between variables concerned with this conflict. If no order is imposed, there may be significant choices for the variable that will represent the nogood conclusion. The approach performance depends mainly on the quality of this choice, which is not covered in PDB.

Generally, for two given variables, the order is not defined at the beginning. There is a partial order that is built during the search process, to achieve the solution if it exists, or to detect the unsatisfiability through the empty nogood.

The partial order between variables is imposed by saved nogoods and safety conditions, a data structure of orders presented by the deleted nogoods.

Definition 4 (Safety condition). A safety condition [11] is defined as an assertion of the form: $x<y$, with $x$ and $y$ variables.

If $S$ is a set of safety conditions, we denote by $\leq_{s}$ the transitive closure of $<$, meaning that $S$ is acyclic if $\leq_{s}$ is anti-symmetric:

$$
x<_{s} y \quad \Rightarrow \quad x \leq_{s} y \quad \& \quad y \not \leq_{s} x .
$$

In another way, $x<_{s} y$ if there is a sequence (possibly empty) of safety conditions:

$$
x<z_{1}<z_{2}<\cdots<z_{n}<y \text {. }
$$

The first improvement of Extended Partial-order Dynamic Backtracking (EPDB) [2] is to extend the classical description of PDB, by exploiting its flexibility to repair assignments using ordering heuristics to control changes. Thus, in a conflict situation, when no order exists between variables in conflict, EPDB changes the less relevant sub-problem. Otherwise, it keeps as long as possible the most difficult sub-problems intact, in order to avoid falling into more complicated conflicts. Technically, this is equivalent to attribute the variable in the most complex sub-problem to the nogood conclusion, whenever a conflict has to be justified, or a nogood resolution occurs due to a dead-end. The usefulness of variable ordering heuristics (VOHs) appears in these two situations. 
The second improvement of EPDB is to satisfy the current constraint $C_{i}$ before selecting another one to verify. In fact, when a checked constraint $C_{i}$ is violated, PDB [11] changes the value of a $C_{i}$ concerned variable and another constraint is checked. The satisfaction of $C_{i}$ is not insured, but it will be re-checked later.

To reuse solution and past reasoning techniques, EPDB (Figure 1) starts the search with a DCSP that contains a full assignment (e.g. past solution) keeping retroactive data structures of nogoods ngList saved during the past search process.

EPDB updates the set of constraints cstrs which need to be verified and satisfied; it clears the safety conditions list SC, which contains the old orders expressed by deleted nogoods, and initiates the information of the solution obtainment and the empty nogood at False. Then, it checks the consistency of every constraint till finding a solution or generating the empty nogood (lines 1-7).

If an inconsistency is detected, a new nogood ng is generated using Generate $N g$ (lines 7-9). The function is called to determine the variable, among the variables in conflicts conflictVars, which will represent the right-hand side (Rhs) of the nogood. It generates the nogood respecting the existing order, which is expressed by both of the list of nogoods ngList and safety conditions $S C$ (lines 30-32), or according to the chosen variable ordering heuristic $V O H$ if no order exists (line 33). Next, the new nogood is added into the list of nogoods ngList, and Repair, the recursive function, is called, in order to find a new assignment to $R h s(n g)$, the right-hand side variable (the conclusion) of $n g$ (lines 10 and 11).

If the constraint $c$ is consistent, it is moved from the DCSP constraints set cstrs to freeCstrs, which saves all checked and satisfied constraints of the DCSP. Then, another constraint is chosen to be checked (lines 12-14). If all constraints are satisfied (DCSP.cstrs is empty), the solution is found, and the search is ended (lines 15-17).

As already said, the function Repair looks for an assignment to its parameter var, therefore, it verifies its domain (line 18). If the function cannot assign a value to var due to a domain wipe out, it builds a nogood $n g 2$, which is the resolution nogood of all nogoods where var appears in the conclusion, using the procedure Resolution $\mathrm{Ng}$ (lines 18 and 19). If the resolved nogood is empty, no solution can be found, thus, the search is ended (lines 20-22). Else, Repair is called recursively to look for a new assignment to Rhs(ng2), the right-hand side of the nogood $n g 2$. If $R h s(n g 2)$ cannot change its value due to a domain wipe out, an empty nogood is generated. The function returns False due to the solution's non-existence (lines 23 and 24). Otherwise, Repair deletes all nogoods where var exists in the antecedent and updates the safety conditions list $S C$ (lines 25 and 26). Updating $S C$ consists of deleting from it all orders where the right-hand side is equal to the right-hand side of any of deleted nogoods, before preserving orders imposed by them in $S C$. Then, it updates the set of constraints by moving to cstrs the constraints involving var and associates to it a new value from its valid domain (lines 27-28).

Resolution $N g$ is called when the variable var detects a dead-end. Thus, the procedure stores, in a structure conflictVars, all variables appearing on the left- 


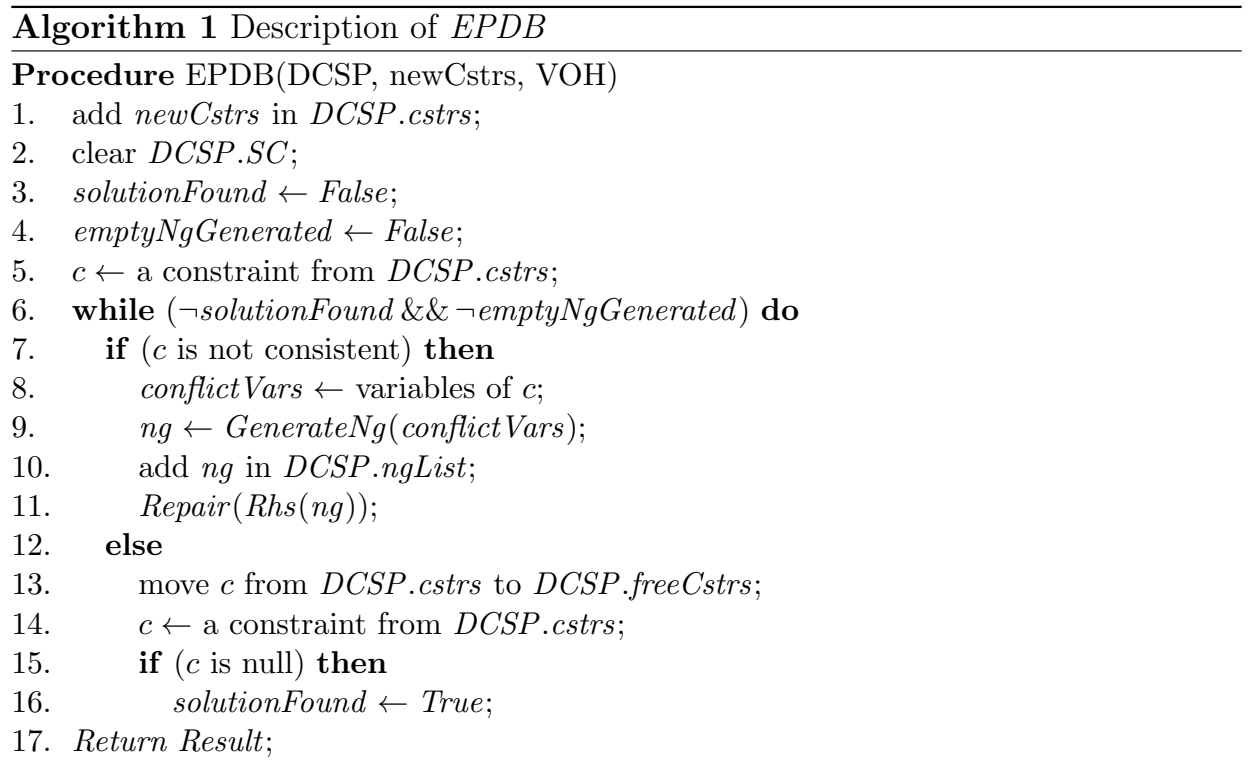

Function Repair(var)

18. if (domain of var is empty) then

19. $\quad n g 2 \leftarrow$ ResolutionNg(var);

20. if ( $n g 2$ is empty) then

21. $\quad$ emptyNgGenerated $\leftarrow$ True;

22. Return False;

23. if $(\neg \operatorname{Repair}(\operatorname{Rhs}(n g 2)))$ then

24. Return False;

25. remove from DCSP.ngList nogoods as var in Lhs;

26. update DCSP.SC according to deleted nogoods;

27. move var's constraints from DCSP.freeCstrs to DCSP.cstrs;

28. change value of var;

29. Return True;

Function GenerateNg(conflictVars)

30. lower $\leftarrow$ get lower priority in conflictVars;

31. if (lower is not null) then

32. Return nogood between conflictVars where lower is Rhs;

33. Return nogood between conflictVars according $\mathrm{VOH}$;

Function ResolutionNg(var)

34. conflictVars $\leftarrow$ variables in Lhs of nogoods prohibiting values of var;

35. GenerateNg(conflictVars); 
hand side (Lhs) of all nogoods prohibiting var's values (line 34). Then, it calls GenerateNg to generate the relevant nogood (line 35).

\subsection{Profound Degree Heuristic}

When a constraint is violated, a concerned variable has to change the value. This change can affect neighbors, hence the interest of deg heuristic to choose the variable to change.

In fact, when a variable changes the value, the change may affect, in addition to its neighbors, all of the network variables, with a given probability.

The goal of profound degree (pdeg) [1] is to change, when a conflict is detected, the concerned variable that can affect the network the least possible. Therefore, pdeg of each variable estimates its influence relatively to its position in the network. This is performed at the beginning of the search. The influence estimation considers the whole network constraints, and associates to each constraint a specific weight, higher or lower, in relation to its distance from the concerned variable. pdeg is considered as the sum of these weights.

\section{LAZY REPAIRING BACKTRACKING}

\subsection{Motivation}

EPDB has the advantage to be largely flexible compared to PDB: it exploits the flexibility of PDB to build optimal orders between variables, using VOHs, in order to converge to the termination with minimal checks of constraints.

The drawback of both EBDP and PDB is the verification of the current order between concerned variables before generating a nogood, in conflict or domain wipeout situations. This verification of orders, which are saved in safety conditions and nogoods, is necessary to avoid looping, but it often consumes a significant time, since in most cases, orders are not directly deduced.

To clarify, suppose the next example:

EPDB is used to repair a dynamic CSP. The current constraint to check is $C_{12}$, which is violated. A nogood concerning $X_{1}$ and $X_{2}$ must be generated, thus, the current order between $X_{1}$ and $X_{2}$ is verified.

The actual orders are shown in Table 1 .

\begin{tabular}{|l|l|}
\hline Safety Conditions & Nogoods \\
\hline$X_{2}<X_{i}(1)$ & $X_{k}=v_{k} \Longrightarrow X_{l} \neq v_{l}(4)$ \\
$X_{i}<X_{m}(2)$ & $X_{k}=v_{k} \Longrightarrow X_{1} \neq v_{1}(5)$ \\
$X_{j}<X_{k}(3)$ & $X_{i}=v_{i} \Longrightarrow X_{j} \neq v_{j}(6)$ \\
\hline
\end{tabular}

Table 1. Existing orders

In fact, $X_{2}<X_{1}$, since $X_{2}<X_{i}(1), X_{i}<X_{j}(6), X_{j}<X_{k}(3)$ and $X_{k}<X_{1}(5)$. But deducing the order between $X_{1}$ and $X_{2}$ is not directly done, as the variables 
involved in this order, namely $X_{2}, X_{i}, X_{j}, X_{k}$ and $X_{1}$, are involved in other nogoods and safety conditions which are stored before the concerned orders. $X_{i}$ is involved in (2) which is placed before (6) and $X_{k}$ in (4) which is before (5), then, (2) and (4) are also verified.

Moreover, determining order takes an expensive time also when no order exists between variables in conflict. Unfortunately, this must be proven by verifying all nogoods and safety conditions concerning them directly (orders where they appear) and indirectly (orders where their neighbours appear directly and indirectly).

As an example, suppose once again using EPDB to repair a DCSP, such as the current constraint to check $C_{12}$ is violated. To generate a nogood, the current order between $X_{1}$ and $X_{2}$ is verified.

The actual orders are shown in Table 2

\begin{tabular}{|l|l|}
\hline Safety Conditions & Nogoods \\
\hline$X_{2}<X_{i}(1)$ & $X_{k}=v_{k} \Longrightarrow X_{l} \neq v_{l}(4)$ \\
$X_{i}<X_{m}(2)$ & $X_{1}=v_{1} \Longrightarrow X_{k} \neq v_{k}(5)$ \\
$X_{j}<X_{n}(3)$ & \\
\hline
\end{tabular}

Table 2. Existing orders

The approach checks (1) then (2) to test if $X_{2}$ precedes $X_{1}$, and (5) then (4) to test if $X_{1}$ precedes $X_{2}$, before concluding that no order exists between them, and establishing one using the specified $\mathrm{VOH}$.

Therefore, in Lazy Repairing Backtracking (LRB), orders are deduced directly using a VOH. Thus, while safety conditions in EPDB are only used to store orders expressed by the removed nogoods, and nogoods are also used to save justifications of prohibitions (to avoid choosing a value which will cause an already met conflict and to restore values when causes are no longer valid), safety conditions are rejected and only nogoods are kept.

The only issue that can exist, when establishing the order directly using a $\mathrm{VOH}$, i.e. without verifying the existence of order between concerned variables, is the termination when the VOHs values change. To remedy this problem, only static VOHs are used, in order to have a fixed order between variables during the search process, especially since the static VOHs, deg and pdeg, have the best behavior in repairing [1, 2].

\subsection{Behavior of LRB Compared to PDB and EPDB}

As shown in Figure 1, after checking a constraint $C_{i j}$, satisfied or not, PDB checks another one $C_{k l}$. In fact, if $C_{i j}$ is violated, PDB tests if an order already exists between the concerned variables $i$ and $j$. Next, it builds a nogood ng, respecting the order, if there exist. If no order exists between $i$ and $j$, it builds any nogood ng which will impose a new order between $i$ and $j$. Then, the conclusion of ng changes the value and another constraint $C_{k l}$ is checked. 


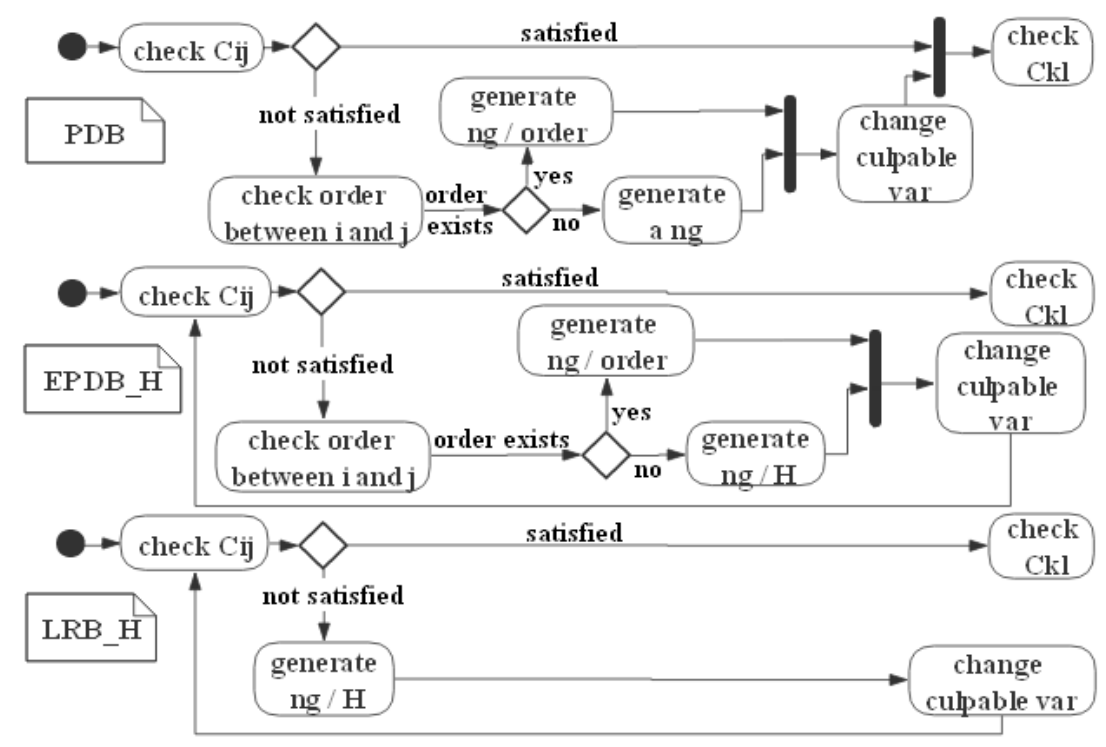

Figure 1. Behaviour of PDB, EPDB and LRB

After checking a constraint $C_{i j}$, EPDB checks another one $C_{k l}$ only if $C_{i j}$ is satisfied. If $C_{i j}$ is violated, it tests if an order already exists between the concerned variables $i$ and $j$. Next, it builds a nogood ng, respecting the order if there exists. If no order exists between $i$ and $j$, the nogood respects the chosen heuristic $H$. Then, the conclusion of ng changes the value, and $C_{i j}$ is re-checked.

LRB, like EPDB, after checking a constraint $C_{i j}$, checks another one $C_{k l}$ only if $C_{i j}$ is satisfied. Otherwise, LRB builds a nogood ng, respecting the chosen heuristic $H$. Then, the conclusion of ng changes the value, and $C_{i j}$ is checked again.

\subsection{An Example Run}

Let us consider the CSP illustrated in Figure 2,

- $X=\{X 1, X 2, X 3, X 4\}$.

- $D=\left\{D_{1}, D_{2}, D_{3}, D_{4}\right\}$ such as $D_{i}=\{1,2,3\}, \forall i \in[1,4]$.

- $C=\{X 1<X 2, X 1 \neq X 4, X 2=X 3, X 2 \neq X 4\}$.

All variables are assigned by the value 1 . For simplicity, we consider that this first assignment (before perturbation) was found using a simple resolution approach (FC for example), then, at the beginning of repairing, no nogood or safety condition are saved in the last search. Thus, no order is imposed between variables. 


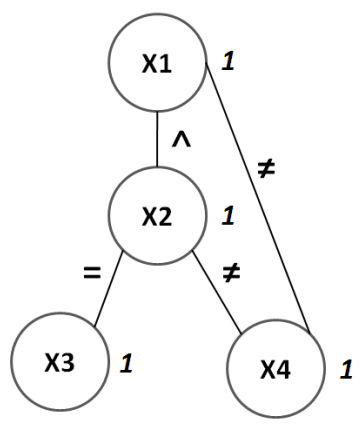

Figure 2. A constraint network example

Table 3 describes EPDB using the deg heuristic. The approach calculates, before starting the search, deg of each variable in the network: $\operatorname{deg}(X 1)=2, \operatorname{deg}(X 2)=3$, $\operatorname{deg}(X 3)=1$ and $\operatorname{deg}(X 4)=2$.

All variables have the same assigned value 1 . This assignment violates more than one constraint. A constraint ordering heuristic [12] can be used to improve the search, but, in order to treat different cases, we assume that the constraints are ordered as shown in to check column.

The first checked constraint checked is the one between $X 1$ and $X 2$ (line 1). This constraint is violated, and as no order exists yet between $X 1$ and $X 2$ (add column is empty), the heuristic value of $X 1$ and $X 2$ is verified to determine the order. $\operatorname{deg}(X 1)<\operatorname{deg}(X 2)$, thus, the generated nogood is (1). $X 1$ changes the value and the constraint is re-checked before selecting another one (line 2). $C_{12}$ is not consistent, then, the existence of order between $X 1$ and $X 2$ is tested before generating the nogood. (1) is tested then (2) is generated. $X 1$ changes the value and the constraint, which is still not consistent, is re-checked (line 3 ). To generate the nogood, the existence of order between $X 1$ and $X 2$ is tested. Then, (1) is tested and (3) is generated. $X 1$ has no more values in its domain, then, EPDB resolves (1), (2) and (3) as (4), removes the three no valid nogoods (line 4), saves their expressed order as a safety condition (5) and changes the value of $X 2$, the unique cause of the domain wipeout of $X 1$ (line 5). $X 1$ is assigned to its first valid value, and the constraint, which is consistent, is rechecked (line 6). As $C_{12}$ is satisfied, it is moved to the list of free constraints free cstrs, which contains all tested and satisfied constraints, and the next constraint to verify is checked. $C_{14}$ is violated (line 7). The existence of order between $X 1$ and $X 4$ is tested, but no order in add contains neither $X 1$ nor $X 4$. Then, the heuristic value is verified. As $\operatorname{deg}(X 1)=\operatorname{deg}(X 4)$, any order is chosen, we assume that in similar cases, the chosen order is the lexicographic one. Thus, the nogood is generated as (6). $X 4$ changes the value, then, $C_{14}$ is consistent (line 8 ) and it is moved to free cstrs. The next constraint to check is $C_{24}$ which is violated (line 9), (5) and (6) are tested, then, (7) is generated, the value of $X 4$ is changed (line 10) and $C_{14}$, the only constraint 
in free cstrs concerning $X 4$, is moved to to check list. The next checked constraint is $C_{23}$ which is violated (line 11), the tested orders are (5) then (6), and (7). As no order exists between the two variables, the chosen heuristic is used to generate (8), then, the value of $X 3$ is changed (line 12). $C_{23}$ is consistent, thus, it is moved to free cstrs. $C_{14}$ is then checked (line 13), it is satisfied so it is moved to free cstrs. to check is empty (line 14), thus, all constraints are satisfied and the solution is found.

\begin{tabular}{|c|c|c|c|c|c|c|c|}
\hline & to check & free cstrs & $\mathbf{X}_{1} \mathbf{X}_{2} \quad \mathbf{X}_{3} \mathbf{X}_{4}$ & checked & test & add & remove \\
\hline 1 & $C_{12} C_{14} C_{24} C_{23}$ & & $1,1,1,1$ & $C_{12} \times$ & deg & $X_{2}=1 \Rightarrow X_{1} \neq 1(1)$ & \\
\hline 2 & $C_{12} C_{14} C_{24} C_{23}$ & & $2,1,1,1$ & $C_{12} \times$ & (1) & $X_{2}=1 \Rightarrow X_{1} \neq 2(2)$ & \\
\hline 3 & $C_{12} C_{14} C_{24} C_{23}$ & & $3,1,1,1$ & $C_{12} \times$ & (1) & $X_{2}=1 \Rightarrow X_{1} \neq 3(3)$ & \\
\hline 4 & $C_{12} C_{14} C_{24} C_{23}$ & & $-, 1,1,1$ & & & $X_{2} \neq 1(4)$ & $(1),(2),(3)$ \\
\hline 5 & $C_{12} C_{14} C_{24} C_{23}$ & & $-, 2,1,1$ & & & $X_{2}<X_{1}(5)$ & \\
\hline 6 & $C_{12} C_{14} C_{24} C_{23}$ & & $1,2,1,1$ & $C_{12} \sqrt{ }$ & & & \\
\hline 7 & $C_{14} C_{24} C_{23}$ & $C_{12}$ & $1,2,1,1$ & $C_{14} \times$ & deg & $X_{1}=1 \Rightarrow X_{4} \neq 1(6)$ & \\
\hline 8 & $C_{14} C_{24} C_{23}$ & $C_{12}$ & $1,2,1,2$ & $C_{14} \sqrt{ }$ & & & \\
\hline 9 & $C_{24} C_{23}$ & $C_{12} C_{14}$ & $1,2,1,2$ & $C_{24} \times$ & $(5),(6)$ & $X_{2}=2 \Rightarrow X_{4} \neq 2(7)$ & \\
\hline 10 & $C_{24} C_{23} C_{14}$ & $C_{12}$ & $1,2,1,3$ & $C_{24} \sqrt{ }$ & & & \\
\hline 11 & $C_{23} C_{14}$ & $C_{12} C_{24}$ & $1,2,1,3$ & $C_{23} \times$ & $(5),(6),(7), \operatorname{deg}$ & $X_{2}=2 \Rightarrow X_{3} \neq 1(8)$ & \\
\hline 12 & $C_{23} C_{14}$ & $C_{12} C_{24}$ & $1,2,2,3$ & $C_{23} \sqrt{ }$ & & & \\
\hline 13 & $C_{14}$ & $C_{12} C_{24} C_{23}$ & $1,2,2,3$ & $C_{14} \sqrt{ }$ & & & \\
\hline 14 & & $C_{12} C_{24} C_{23} C_{14}$ & $1,2,2,3$ & NULL & & & \\
\hline
\end{tabular}

Table 3. Execution of $E P D B_{\text {deg }}$

Similarly to EPDB, when LRB is executed using the same heuristic deg, the approach calculates, before starting the search, deg of all network variables.

It proceeds like EPDB with the following differences:

- to generate a nogood, LRB tests directly the chosen VOH values. Then, in test column, each constraint violation (nogood to generate) causes only one test, which is the test of deg, contrary to EPDB (the test column of Table 3).

- LRB does not need the information about the order saved in safety conditions, since the order is determined by the used $\mathrm{VOH}$. Then, when a nogood is removed, no safety condition is retained (in Table 3, after removing nogoods in remove column of line 4 , add column of line 5 will be empty).

\subsection{Description of the Proposed Approach}

To reuse the solution and the past reasoning techniques, LRB starts the search with a DCSP that contains the past solution, keeping retroactive data structures of nogoods ngList saved during the past search process.

LRB proceeds exactly like EPDB, with the following major differences:

- to generate a nogood when a conflict is detected, LRB does not verify the order between concerned variables as EPDB does. Then, in line 9 of Figure 1, the chosen $\mathrm{VOH}$ is directly used to establish the order.

- similarly, to generate the resolution nogood when a domain wipe-out is produced, LRB does not verify the order between the responsible variables as EPDB does. 
Thus, in line 35 of Figure 1, the chosen VOH is directly used to generate the nogood.

- As no order verification will be required, LRB does not use the safety condition list to store orders expressed by the removed nogoods, then, lines 2 and 26 in Figure 1] are removed.

\subsection{Termination Proof}

It is proven in [6] that PDB terminates. In fact, PDB respects the relative ordering conditions corresponding to the ordered nogoods. Moreover, it imposes safety conditions, which are relative ordering conditions, in order to retain some of the ordering conditions of the nogoods that have been removed. The order selected for new nogoods is required to respect both types of ordering conditions. Thus, the resulting set of conditions could not be cyclic.

LRB proceeds in the same manner of PDB, but drops safety conditions. To guarantee termination, it uses only static VOHs and is based on the used SVOH for the selected order.

\section{EXPERIMENTAL STUDY}

\subsection{Experiments Concerning Repairing}

In this section, we compare LRB to EPDB, to show the improvement of the new approach. We also compare it to MAC-2001 [4, 5], to identify the maximal rate of perturbation that tolerates repairing, i.e. from which rate of disturbance the use of a good resolution algorithm is more efficient to deal DCSPs.

Some integrations of MAC in intelligent backtrackers were already proposed [16, 13], but we choose MAC-2001 as we judge it to be one of the most efficient resolving CSPs strategies while being the most familiar constraint propagation one.

Heuristics used in LRB and EPDB are pdeg and deg. To prove that performance of LRB is due essentially to a well-chosen heuristic, we use also the lexicographic order heuristic, which is used instead of the arbitrary order, as LRB supports only the static ordering to avoid looping. MAC-2001 is combined to the dom/deg heuristic like in [14. We note that the arc consistency strategy can be also improved by using dom/wdeg [7], but as shown in [15], this heuristic is efficient essentially in combination with AC-3.

As evaluation criteria, we measure the runtime and the number of Constraints Checks (CCs).

\subsubsection{Experiments on Uniform Random Binary DCSP}

A uniform random binary DCSP $P$ is a sequence of static CSPs which are subject to perturbations. 
CSPs are characterized by $\left\langle n, d, p_{1}, p_{2}\right\rangle$, where $n$ is the number of variables, $d$ the number of values per variable, $p_{1}$ the network connectivity, defined as the ratio of existing binary constraints to possible binary constraints, and $p_{2}$ the constraints tightness, that represents the proportion of forbidden pairs among the constraints.

Tests are performed on sparse and dense problems, respectively $\langle 30,10,0.25$, $0.45\rangle$ and $\langle 30,10,0.75,0.19\rangle$. The chosen problems are the satisfiable ones at the transition phase [8] (the most complicated area).

For each pair of fixed density and constraints tightness $\left\langle p_{1}, p_{2}\right\rangle, 30$ different instances are treated by each algorithm and the present results are the averages of these 30 runs.

The rate of injected constraints $\% I C=\frac{\mid \text { new constraints } \mid}{\mid \text { whole problem constraints } \mid}$. It indicates the rate of disturbance that will be treated using both LRB and EPDB. IC varies from $1 \%$ to $100 \%$, such as for each given disturbance of $d \%$, the added constraints are the same as those in the disturbance of $d-1 \%$, plus $1 \%$ more constraints. The 30 final problems (after disturbance) are the same for each rate of disturbance.

For DCSP approaches, namely LRB and EPDB, the computed effort is the one for repairing the past solution, i.e. which existed before disturbing.

As $p_{2}$ is known, we tried to replace, in the pdeg calculation, the parameter $1 / 2$ by $p_{2}$. But results were not significantly better than those of using $1 / 2$, then, we choose to keep the parameter $1 / 2$ for all kinds of problems. We fix the time-out at 7 minutes. Experiments are performed on the Java platform, on a core i7 PC (3.6 GHz processor and $16 \mathrm{~GB}$ RAM).

Concerning the number of CCs $\left(2^{\text {nd }}\right.$ part of Tables 4 and 5), using LRB with a $\mathrm{VOH}$ is almost the same as using EPDB with the same $\mathrm{VOH}$. But regarding the execution time $\left(1^{\text {st }}\right.$ part of Tables 4 and 5 ), LRB exceeds EPDB very remarkably, regardless of the used $\mathrm{VOH}$.

Excepting the small disturbances, MAC-2001 often produces less CCs than LRB ( $2^{\text {nd }}$ part of Tables 4 and 5), mainly when LRB uses the deg heuristic.

From a certain small disturbance $(13 \%$ for sparse problems and $10 \%$ for those dense), LRB needs almost the same effort to repair (Tables 4 and 5), whatever the rate of injected constraints IC. This effort is not stable, regardless of the used heuristic, but it is subject to same sudden falls.

Regarding LRB, the use of pdeg is often more beneficial than using deg.

$L R B_{p d e g}$ is always faster than MAC-2001 ( $1^{\text {st }}$ part of Tables 4 and 5), even disturbing $100 \%$ of constraints, which is equivalent to resolving the whole problems. $L R B_{\text {deg }}$ is sometimes less efficient than MAC-2001, but it is anyway a good concurrent.

\subsubsection{Experiments on Meetings Scheduling Problems}

A Dynamic Meeting Scheduling Problem (DMSP) is a sequence of static Meeting Scheduling Problems (MSPs): $M S P_{0}, M S P_{1}, \ldots, M S P_{\alpha-1}, M S P_{\alpha}, \ldots$, where the network is subject to constraints perturbations, and each $M S P_{i}$ differs from the latest one $M S P_{i-1}$ by the addition of some constraints. 


\begin{tabular}{|c|c|c|c|c|c|c|c|}
\hline$\% \mathrm{IC}$ & MAC-2001 & $L R B_{\text {pdeg }}$ & $L R B_{\text {deg }}$ & $L R B_{\text {lex }}$ & $E P D B_{p d e g}$ & $E P D B_{d e g}$ & \\
\hline 1 & \multirow{23}{*}{0.094} & 0.006 & 0.008 & 1.471 & 0.508 & 0.723 & \multirow{23}{*}{ time(sc) } \\
\hline 4 & & 0.024 & 0.026 & 1.835 & 2.242 & 2.662 & \\
\hline 7 & & 0.048 & 0.065 & 2.047 & 4.842 & 6.254 & \\
\hline 10 & & 0.059 & 0.073 & 2.304 & 5.788 & 7.047 & \\
\hline 13 & & 0.079 & 0.089 & 2.650 & 7.517 & 8.373 & \\
\hline 16 & & 0.082 & 0.093 & 2.860 & 8.081 & 9.338 & \\
\hline 19 & & 0.082 & 0.097 & 2.809 & 8.099 & 9.243 & \\
\hline 22 & & 0.085 & 0.094 & 2.876 & 8.214 & 9.479 & \\
\hline 25 & & 0.080 & 0.095 & 2.878 & 7.759 & 9.479 & \\
\hline 28 & & 0.080 & 0.099 & 2.860 & 8.020 & 9.467 & \\
\hline 31 & & 0.078 & 0.101 & 2.891 & 7.263 & 8.093 & \\
\hline 34 & & 0.075 & 0.102 & 2.881 & 7.330 & 8.402 & \\
\hline 40 & & 0.062 & 0.075 & 2.869 & 8.321 & 8.069 & \\
\hline 46 & & 0.069 & 0.078 & 2.893 & 9.521 & 10.455 & \\
\hline 52 & & 0.080 & 0.093 & 2.894 & 8.919 & 10.799 & \\
\hline 58 & & 0.065 & 0.076 & 2.914 & 8.897 & 8.989 & \\
\hline 64 & & 0.075 & 0.084 & 2.942 & 8.593 & 8.258 & \\
\hline 70 & & 0.082 & 0.097 & 2.932 & 7.678 & 9.113 & \\
\hline 76 & & 0.073 & 0.106 & 2.871 & 8.189 & 10.227 & \\
\hline 82 & & 0.085 & 0.099 & 2.903 & 8.266 & 10.381 & \\
\hline 88 & & 0.083 & 0.100 & 2.889 & 8.203 & 10.337 & \\
\hline 94 & & 0.085 & 0.099 & 2.897 & 8.510 & 10.315 & \\
\hline 100 & & 0.084 & 0.099 & 2.868 & 8.230 & 9.888 & \\
\hline 1 & \multirow{4}{*}{169168} & 15108 & 20199 & 3032339 & 15108 & 20199 & \multirow{23}{*}{$\mathrm{CCs}$} \\
\hline 4 & & 58651 & 65202 & 3806153 & 59779 & 68687 & \\
\hline 7 & & 117728 & 157599 & 4227876 & 123667 & 157298 & \\
\hline 10 & & 145623 & 181242 & 4764235 & 148286 & 180593 & \\
\hline 13 & \multirow{8}{*}{169168} & 192355 & 214418 & 5527584 & 189534 & 209805 & \\
\hline 16 & & 201644 & 225471 & 5992181 & 205562 & 230519 & \\
\hline 19 & & 201099 & 235586 & 5879701 & 204280 & 228941 & \\
\hline 22 & & 207509 & 230595 & 6049455 & 206419 & 233566 & \\
\hline 25 & & 196193 & 233567 & 6047661 & 197054 & 235723 & \\
\hline 28 & & 196130 & 243182 & 6001166 & 203289 & 237828 & \\
\hline 31 & & 191143 & 247404 & 6060702 & 185955 & 206190 & \\
\hline 34 & & 183019 & 248225 & 6058750 & 188308 & 211733 & \\
\hline 40 & 169168 & 151765 & 185843 & 6015178 & 212178 & 202816 & \\
\hline 46 & \multirow{2}{*}{169168} & 170804 & 192706 & 6066024 & 242173 & 261430 & \\
\hline 52 & & 197833 & 226562 & 6076485 & 228440 & 268696 & \\
\hline 58 & 169168 & 161864 & 187689 & 6094090 & 227370 & 228662 & \\
\hline 64 & \multirow{7}{*}{169168} & 186754 & 207362 & 6138907 & 223674 & 210593 & \\
\hline 70 & & 200774 & 237995 & 6029206 & 195718 & 229670 & \\
\hline 76 & & 181101 & 255407 & 6029857 & 213139 & 257020 & \\
\hline 82 & & 207836 & 246870 & 6084857 & 210158 & 259925 & \\
\hline 88 & & 201640 & 248743 & 6070954 & 210870 & 259146 & \\
\hline 94 & & 207763 & 243764 & 6068184 & 217752 & 257468 & \\
\hline 100 & & 206479 & 241355 & 6014275 & 207955 & 246269 & \\
\hline
\end{tabular}

Table 4. Execution time by seconds and Number of CCs $\left(p_{1}=0.25, p_{2}=0.45\right)$ 


\begin{tabular}{|c|c|c|c|c|c|c|c|}
\hline$\% \mathrm{IC}$ & MAC-2001 & $L R B_{\text {pdeg }}$ & $L R B_{d e g}$ & $L R B_{\text {lex }}$ & $E P D B_{\text {pdeg }}$ & $E P D B_{d e g}$ & \\
\hline 1 & \multirow{23}{*}{11.054} & 0.698 & 0.896 & 1.874 & 111.769 & 194.669 & \multirow{23}{*}{ time (sc) } \\
\hline 4 & & 3.328 & 4.565 & 13.510 & 370.385 & - & \\
\hline 7 & & 4.698 & 5.877 & 32.571 & - & - & \\
\hline 10 & & 6.590 & 7.427 & 40.478 & - & - & \\
\hline 13 & & 7.379 & 6.675 & 42.993 & - & - & \\
\hline 16 & & 7.657 & 7.591 & 44.719 & - & - & \\
\hline 19 & & 7.847 & 8.638 & 44.873 & - & - & \\
\hline 22 & & 8.835 & 9.253 & 44.903 & - & - & \\
\hline 25 & & 5.962 & 8.694 & 44.027 & - & - & \\
\hline 28 & & 7.711 & 9.874 & 44.113 & - & - & \\
\hline 31 & & 7.848 & 9.780 & 44.086 & - & - & \\
\hline 34 & & 8.938 & 10.503 & 44.004 & - & - & \\
\hline 40 & & 8.357 & 9.736 & 43.991 & - & - & \\
\hline 46 & & 8.183 & 9.297 & 44.067 & - & - & \\
\hline 52 & & 10.209 & 9.597 & 44.148 & - & - & \\
\hline 58 & & 9.427 & 10.268 & 43.885 & - & - & \\
\hline 64 & & 10.794 & 10.731 & 43.960 & - & - & \\
\hline 70 & & 10.688 & 11.700 & 43.985 & - & - & \\
\hline 76 & & 8.355 & 9.925 & 44.026 & - & - & \\
\hline 82 & & 8.946 & 10.461 & 44.056 & - & - & \\
\hline 88 & & 8.271 & 9.475 & 44.145 & - & - & \\
\hline 94 & & 7.615 & 8.883 & 44.184 & - & - & \\
\hline 100 & & 7.600 & 8.898 & 44.242 & - & - & \\
\hline 1 & \multirow{2}{*}{11091737} & 2053499 & 2676894 & 25300103 & 2063315 & 2687746 & \multirow{23}{*}{$\mathrm{CCs}$} \\
\hline 4 & & 10050253 & 13609155 & 37787281 & 7700192 & - & \\
\hline 7 & \multirow{21}{*}{11091737} & 14196226 & 17492480 & 90474816 & - & - & \\
\hline 10 & & 19917644 & 22168200 & 112775270 & - & - & \\
\hline 13 & & 22323569 & 19950505 & 119366418 & - & - & \\
\hline 16 & & 23178909 & 22661403 & 121865776 & - & - & \\
\hline 19 & & 23692559 & 25854188 & 122555689 & - & - & \\
\hline 22 & & 26684225 & 27586331 & 122734624 & - & - & \\
\hline 25 & & 18061593 & 25937447 & 122755174 & - & - & \\
\hline 28 & & 23355364 & 29466473 & 122739959 & - & - & \\
\hline 31 & & 23793094 & 29203775 & 122837687 & - & - & \\
\hline 34 & & 27114111 & 31384172 & 122669346 & - & - & \\
\hline 40 & & 25268174 & 29127000 & 122734902 & - & - & \\
\hline 46 & & 24710542 & 27748892 & 122643912 & - & - & \\
\hline 52 & & 30820375 & 28720750 & 122943597 & - & - & \\
\hline 58 & & 28428460 & 30704619 & 122704450 & - & - & \\
\hline 64 & & 32524541 & 32018965 & 122798424 & - & - & \\
\hline 70 & & 32150097 & 34963393 & 122669749 & - & - & \\
\hline 76 & & 25192875 & 29655806 & 122768330 & - & - & \\
\hline 82 & & 26922873 & 31354768 & 122696907 & - & - & \\
\hline 88 & & 24982344 & 28307565 & 122684444 & - & - & \\
\hline 94 & & 22954833 & 26581585 & 122766266 & - & - & \\
\hline 100 & & 22965095 & 26579493 & 122848545 & - & - & \\
\hline
\end{tabular}

Table 5. Execution time by seconds and Number of CCs $\left(p_{1}=0.75, p_{2}=0.19\right)$ 
A Meeting Scheduling Problem MSP is characterized by $\langle m, p, n, d, h, t, a\rangle$, where $m$ is the number of meetings, $p$ the number of participants, $n$ the number of meetings per participant and $d$ the number of days. Different time slots are available for each meeting, and $h$ is the number of hours per day, $t$ the duration of the meeting and $a$ the percentage of availability for each participant.

In the used model, $p \times n$ is the number of variables. Variables $X_{i . j}$, such as $i \in p, j \in n$ and $X_{i . j} \in[\operatorname{timeSlot}(1)$, $\operatorname{timeSlot}(m)]$, are the totality of meetings per all participants. Thus, tow types of constraints exist:

- As $p$ is the number of participants and $n$ the number of meetings per participant, for each two distinct variables $X_{i . j}$ and $X_{k . l}$, if $i=k$, then, variables are connected by a constraint termed Arrival Constraint, which means that the participant $i$ must attend two distinct meetings. Therefore, $\left|X_{i . j}-X_{k . l}\right| \geq \delta$, where $\delta$ is the time required to move between $X_{i . j}$ and $X_{k . l}$, expressed in time slots.

- For each two distinct participants $i$ and $k, X_{i . j}=X_{k . l}$ means that $i$ and $k$ must attend the same meeting.

We present our results for the class $\langle 15,30,4,5,10,1,85\rangle$ and vary the rate of disturbance from $\sim 1 \%$ to $\sim 100 \%$. We generated 30 different instances treated by each algorithm and the results are an averages of these 30 runs. As $L R B_{\text {lex }}$ and EPDB are not good concurrent, as shown in the experiments above, we include in comparison only MAC-2001, $L R B_{\text {pdeg }}$ and $L R B_{\text {deg }}$.

Concerning MSPs (Figure 3), $L R B_{\text {pdeg }}$ is more efficient than MAC-2001 regardless of the rate of disturbance, specially respecting the number of CCs. However, MAC-2001 is more effective than $L R B_{\text {deg }}$ respecting runtime (except for a disturbance of $1 \%$ ), even if the rate of CCs is comparable to that of LRB.

\subsection{Experiments Concerning the Problem Solving}

The previous experiments concern satisfiable problems. Experiments on random problems are more precisely focused on satisfiable problems in hard areas of low and high densities. These experiments show that LRB using a VOH, mainly pdeg, is a good concurrent for MAC-2001 although for solving (100\% of IC).

In order to show where MAC-2001 is more efficient than $L R B_{p d e g}$ and $L R B_{d e g}$, we execute the three approaches on random problems, not necessary satisfiable, with the same previous parameters $n, d$ and $p_{1}$. Constraints tightness' vary from $p_{2}=0.1$ to $p_{2}=0.9$. 30 different instances are treated by each algorithm and the present results are the averages of these 30 runs. Experiments are performed on the Java platform, on a Pentium IV PC (2.8 GHz processor and 3.24 GB RAM).

Figures 4 and 5 show that LRB, using either deg or pdeg, is efficient to treat satisfiable problems, i.e. $\left\langle p_{1}=0.25, p_{2} \in\left[0.1,0.45[\rangle\right.\right.$ and $\left\langle p_{1}=0.75, p_{2} \in[0.1,0.19[\rangle\right.$, while MAC-2001 is effective to detect the non satisfiability of problems in $\left\langle p_{1}=\right.$ $\left.\left.\left.0.25, p_{2} \in\right] 0.45,0.9\right]\right\rangle$ and $\left.\left.\left\langle p_{1}=0.75, p_{2} \in\right] 0.19,0.9\right]\right\rangle$. In complex areas, i.e. the 


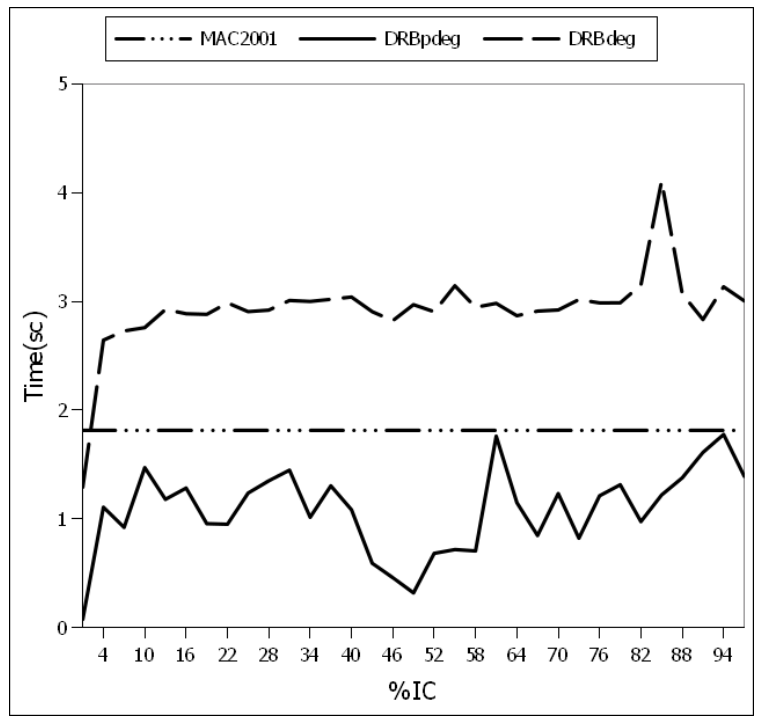

a)

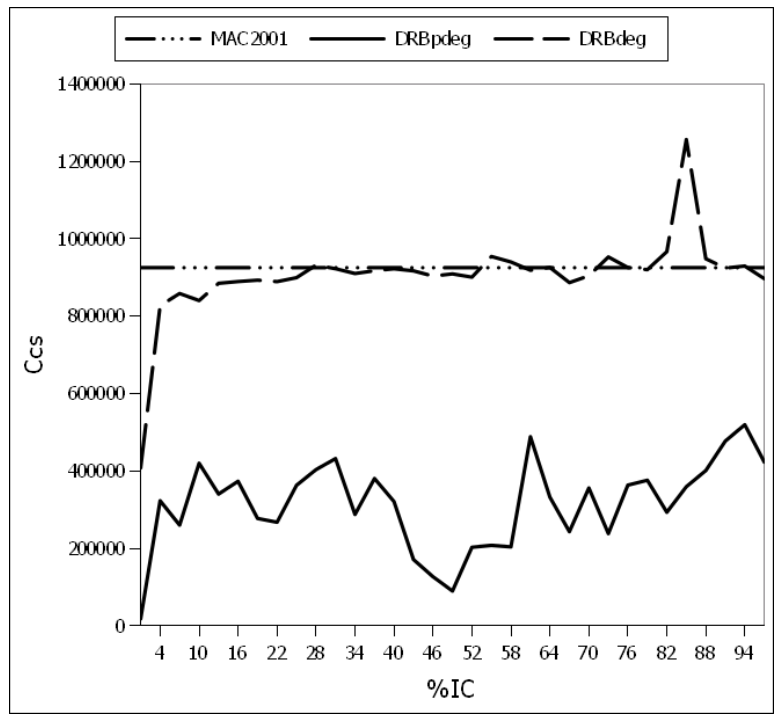

b)

Figure 3. Execution time and Number of CCs for MSPs 


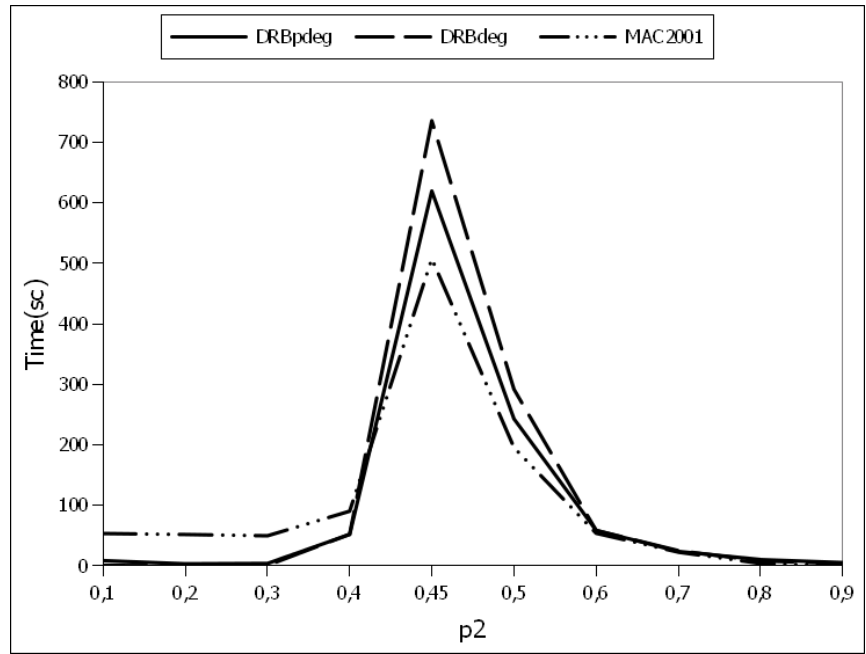

a)

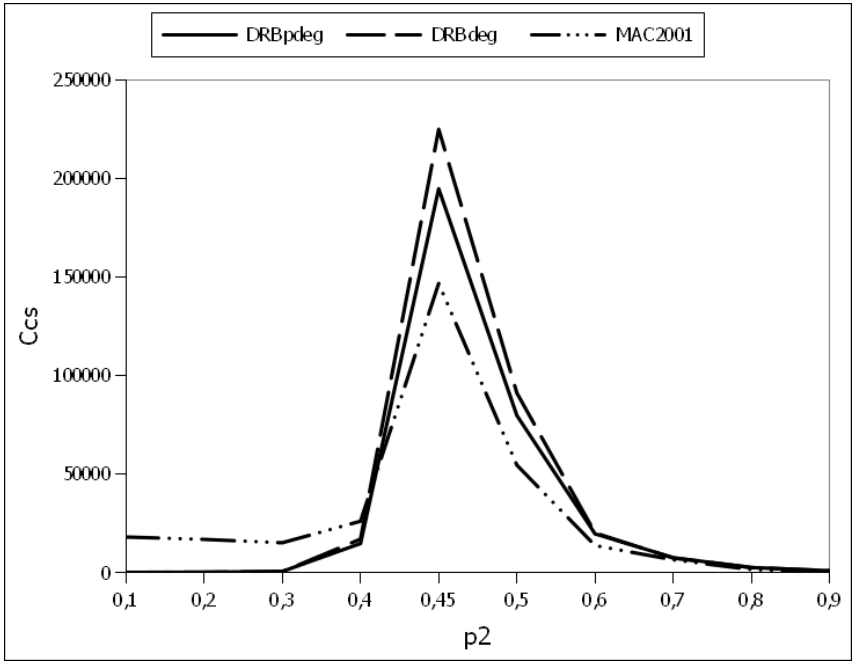

b)

Figure 4. Execution time and Number of CCs for $p_{1}=0.25$ 


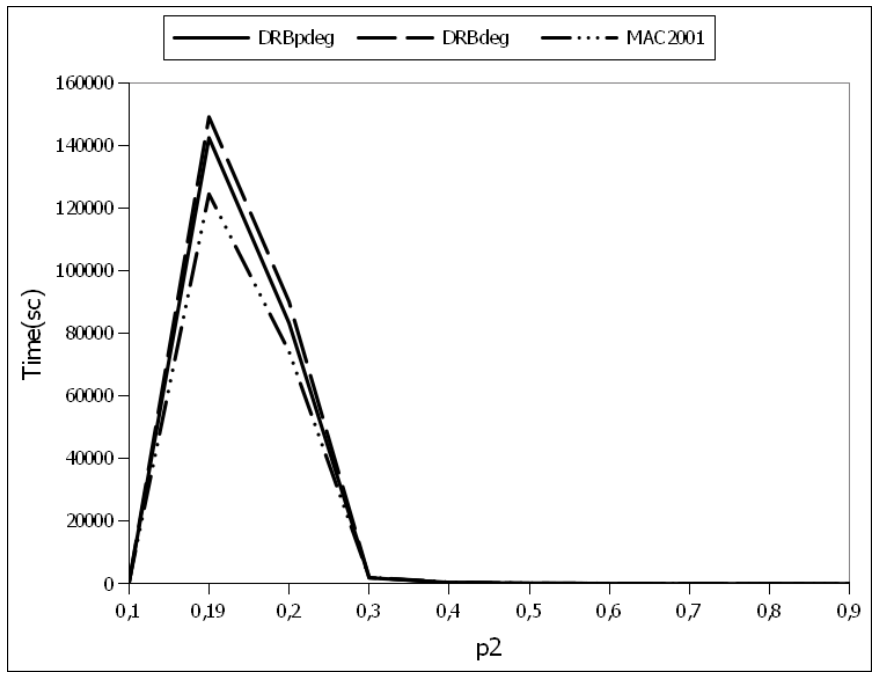

a)

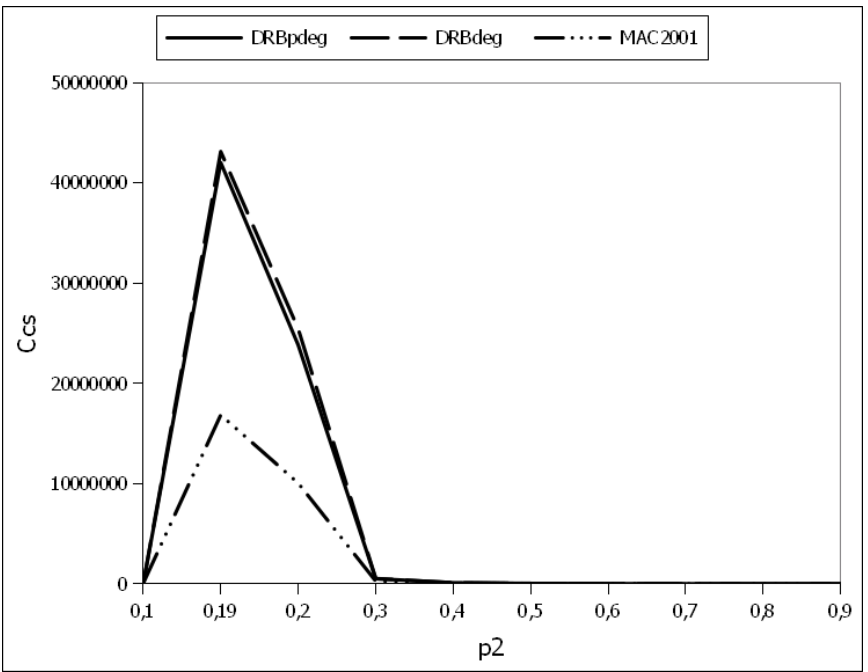

b)

Figure 5. Execution time and Number of CCs for $p_{1}=0.75$ 
transition phases $\left\langle p_{1}=0.25, p_{2}=0.45\right\rangle$ and $\left\langle p_{1}=0.75, p_{2}=0.19\right\rangle$, the majority of problems are non satisfiable, therefore, MAC-2001 is generally better than LRB, mainly for high densities, where satisfiable problems are rare.

For high density problems (Figure 5), using either deg or pdeg in LRB is almost the same.

\section{DISCUSSION}

\subsection{Discussion Concerning Repairing}

As shown in the experiments on satisfiable problems in the transition phases (Tables 4 and 5), LRB is widely more powerful than EPDB in terms of runtime. This is particularly due to avoiding the verification of progressively saved orders, which is done in EPDB each time inconsistency is detected. But regarding CCs, the use of LRB with a VOH is almost the same as using EPDB with the same VOH. Normally, for a fixed heuristic, LRB and EPDB proceed in the same manner, with the difference of testing orders for EPDB. Thus, they must generate the same number of CCs. However, the perturbation, which is a modification in constraints, can change the used heuristic values, as both of the used VOHs deg and pdeg depend on the existing constraints. Then, in EPDB, nogoods stored before disturbance impose an order which is not necessary the same as the one expressed by the present heuristic values, whence the difference of the number of CCs between LRB and EPDB.

From a certain small disturbance, LRB requires almost the same effort to repair, whatever the rate of injected constraints, since problems are chosen from the most complex areas (the transition phases). This effort is not stable, but it is subject to the same sudden falls. In fact, the repairing effort depends on the affectation before disturbance and its consistency with the new constraints. Then, disturbing a problem with some constraints can be easier or harder than disturbing another problem with more constraints, depending on which affectation is satisfiable by more new constraints.

The use of pdeg in LRB (Tables 4 and 5 and Figure 3) is often more effective than using deg, as pdeg estimates the position of each variable regarding the whole network, while deg calculates the position of each variable regarding its neighbourhood.

When problems are hard and satisfiable, LRB, mainly when using pdeg, is a good concurrent of MAC-2001, even for solving (100\% of IC). Nevertheless, when no VOH is used $\left(L R B_{\text {lex }}\right.$ in $1^{\text {st }}$ part of Tables 4 and 5), LRB becomes inefficient comparing to MAC-2001. In fact, the goal of VOHs in repairing is to engender minimal network changes, by keeping the hard sub-problems unchangeable as long as possible, in order to minimize risks of affecting other sub-problems. Therefore, the performance of LRB is also due to the use of a good VOH. 


\subsection{Discussion Concerning Solving}

Concerning problems where constraints tightness is less than the peak (under the transition phase), although injecting the totality of constraints in perturbation, i.e. resolving (Figures 4 and 5), LRB using either deg or pdeg is more efficient than MAC-2001, regardless of problems density. In these areas, most of the problems are satisfiable, thus, the use of arc consistency is not efficient enough. As a matter of fact, the major interest of MAC-2001 is to detect earlier the future domains dead-ends, in order to modify the partial assignments which can not be included in a solution. But in sparse graphs, domains dead-ends are rarely met.

Therefore, for unsatisfying problems, where constraints tightness is higher than the peak, MAC-2001 is more effective than LRB.

On peaks, the majority of problems are unsatisfying, then, MAC-2001 is often more efficient than LRB.

Respecting high densities, $L R B_{\text {pdeg }}$ does not exceed remarkably $L R B_{\text {deg }}$. This is caused by the high connectivity of the network. Therefore, each variable has the majority of other variables as neighbors, then, pdeg is nearly the same as deg.

\section{CONCLUSION AND PERSPECTIVES}

In this paper, we introduce a new approach, namely LRB, which is based on EPDB, to repair the DCSPs solutions rapidly, using static VOHs.

Based on the results of experiments on a variety of problem classes, we show that LRB is a great improvement of EPDB, respecting the runtime, insofar as it avoids the expensive tests of orders.

We have proved that this approach is mainly efficient when using pdeg and that is a good concurrent for MAC-2001. In fact, LRB outperforms MAC-2001 when problems are satisfiable, although using it to solve from the scratch. Thus, LRB has the advantage to be not only destined for DCSPs, but also to CSPs.

We demonstrate that LRB is more effective to treat satisfiable problems while MAC-2001 is more efficient to detect the problem's non-satisfiability. Therefore, for complicated problems where the satisfiability is unknown, the two approaches can be separately launched and the fast resolution is then guaranteed.

As perspectives, we plan to implement a CSP solver, based on LRB, which benefits from LRB advantages and resolves by repairing a complete assignment that violates a minimum number of constraints, in order to converge more rapidly to a solution. We also plan to develop a version of LRB for minimal perturbation problems [19], to preserve DCSPs solutions as much as possible. We also intend to integrate constraint propagation within LRB to benefit from the advantage of the two strategies regardless of the problem's satisfiability. 


\section{REFERENCES}

[1] Benamrane, A.-Acodad, Y.-Benelallam, I.-Bouyakhf, E. H.Bennani Othmani, M.: Modeling Trainings in Faculty of Medicine and Pharmacy of Casablanca as Constraint Satisfaction Problem. The Thirteenth International Workshop on Constraint Modelling and Reformulation, 2014, pp. 17-23.

[2] Zhang, W.-Xing, Z.-Wang, G.-Wittenburg, L.: An Analysis and Application of Distributed Constraint Satisfaction and Optimization Algorithms in Sensor Networks. Proceedings of the Second International Joint Conference on Autonomous Agents and Multiagent Systems (AAMAS '03), Vol. 3, 2003, pp. 185-192, doi: $10.1145 / 860575.860605$.

[3] Verfaillie, G.-Jussien, N.: Constraint Solving in Uncertain and Dynamic Environments: A Survey. Constraints, Vol. 10, 2005, No. 3, pp. 253-281, doi: 10.1007/s10601-005-2239-9

[4] Dechter, R.-Dechter, A.: Belief Maintenance in Dynamic Constraint Networks. Proceedings of the Seventh AAAI National Conference on Artificial Intelligence (AAAI'88), 1988, pp. 37-42.

[5] Ginsberg, M.-McAllester, D.: GSAT and Dynamic Backtracking. In: Borning, A. (Ed.): Principles and Practice of Constraint Programming (PPCP 1994). Springer, Berlin, Heidelberg, Lecture Notes in Computer Science, Vol. 874, 1994, pp. 243-265, doi: 10.1007/3-540-58601-6_105.

[6] Acodad, Y.-Benelallam, I.-Hammoujan, S.-Bouyakhf, E. H.: Extended Partial-Order Dynamic Backtracking Algorithm for Dynamically Changed Environments. 2012 IEEE $24^{\text {th }}$ International Conference on Tools with Artificial Intelligence (ICTAI), Vol. 1, 2012, pp. 580-587, doi: 10.1109/ICTAI.2012.83.

[7] Acodad, Y.-Benamrane, A.-Benelallam, I.-Bouyakhf, E. H.: Profound Degree: A Conservative Heuristic to Repair Dynamic CSPs. In: Iliadis, L., Maglogiannis, I., Papadopoulos, H. (Eds.): Artificial Intelligence Applications and Innovations (AIAI 2014). Springer, Berlin, Heidelberg, IFIP Advances in Information and Communication Technology, Vol. 436, 2014, pp. 140-149, doi: 10.1007/978-3-66244654-6_14.

[8] Bessière, C.-RÉgin, J.-C.: Refining the Basic Constraint Propagation Algorithm. Proceedings of the $17^{\text {th }}$ International Joint Conference on Artificial Intelligence, Vol. 1, 2001, pp. 309-315.

[9] Bessière, C.-RÉgin, J.-C.-Yap, R. H. C.-Zhang, Y.: An Optimal CoarseGrained Arc Consistency Algorithm. Artificial Intelligence, Vol. 165, 2005, pp. 165-185, 2005, doi: 10.1016/j.artint.2005.02.004.

[10] Ginsberg, M. L.: Dynamic Backtracking. Journal of Artificial Intelligence Research, Vol. 1, 1993, No. 1, pp. 25-46, doi: 10.1613/jair.1.

[11] Hammoujan, S.-Acodad, Y.-Benelallam, I.-Bouyakhf, E. H.: Dynamic Constraint Ordering Heuristics for Dynamic CSPs. Applied Mathematical Sciences, Vol. 7, 2013, No. 138, pp. 6889-6907, doi: 10.12988/ams.2013.311657. 
[12] Bliek, C.: Generalizing Partial Order and Dynamic Backtracking. Proceedings of the Fifteenth National/Tenth Conference on Artificial Intelligence/Innovative Applications of Artificial Intelligence (AAAI'98/IAAI'98), 1998, pp. 319-325.

[13] Prosser, P.: MAC-CBJ: Maintaining Arc Consistency with Conflict-Directed Backjumping. Research Report 95/177, Department of Computer Science, University of Strathclyde, Glasgow, 1995.

[14] Jussien, N.-Debruyne, R.-Boizumault, P.: Maintaining Arc-Consistency within Dynamic Backtracking. In: Dechter, R. (Ed.): Principles and Practice of Constraint Programming - CP 2000. Springer, Berlin, Heidelberg, Lecture Notes in Computer Science, Vol. 1894, 2000, pp. 249-261, doi: 10.1007/3-540-45349-0_19.

[15] Mehta, D.- van Dongen, M. R. C.: Reducing Checks and Revisions in CoarseGrained MAC Algorithms. Proceedings of the $19^{\text {th }}$ International Joint Conference on Artificial Intelligence (IJCAI '05), 2005, pp. 236-241.

[16] Boussemart, F.-Hemery, F.-Lecoutre, C.-Sais, L.: Boosting Systematic Search by Weighting Constraints. Proceedings of the $16^{\text {th }}$ European Conference on Artificial Intelligence (ECAI'04), IOS Press, 2004, pp. 146-150.

[17] Mehta, D.-Van Dongen, M. R. C.: Probabilistic Consistency Boosts MAC and SAC. Proceedings of the $20^{\text {th }}$ International Joint Conference on Artificial Intelligence (IJCAI'07), 2007, pp. 143-148.

[18] Crawford, J. M.-Auton, L. D.: Experimental Results on the Crossover Point in Random 3-SAT. Artificial Intelligence, Vol. 81, 1996, No. 1, pp. 31-57, doi: 10.1016/0004-3702(95)00046-1.

[19] Zivan, R.-Grubshtein, A.-Meisels, A.: Hybrid Search for Minimal Perturbation in Dynamic CSPs. Constraints, Vol. 16, 2011, No. 3, pp. 228-249, doi: 10.1007/s10601-011-9108-5

Yosra ACOdAD graduated in 2018 from the Mohammed V University. Her research interests focused on the repair algorithms of satisfaction dynamic constraints problems, one of the popular paradigms of complexe problem-solving at LIMIARF Laboratory under the supervision of Professor El Houssine Bouyakhf and Professor Imade Benelallam. She has published many papers in various international conferences and journals.

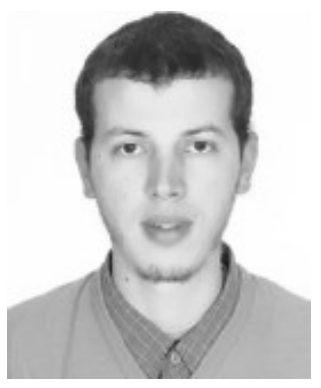

Amine Benamrane is Ph.D. student at the Mohammed V University. His research interests focused on the repair algorithms of satisfaction distributed dynamic constraints problems, a subfield of artificial intelligence. He received his M.Sc. in computer science from the Mohammed V University in Rabat in 2009. Since 2009 , he is an executive at the Scientific Research and Innovation Department of the Higher Education Ministry of Morocco. His work is focused on promoting of research results and boosting collaborations between researchers and companies. In 2014 he began his Ph.D. at LIMIARF Laboratory under the supervision of Professor El Houssine Bouyakhf and Professor Imade Benelallam. He has published papers in various international conferences and journals. 


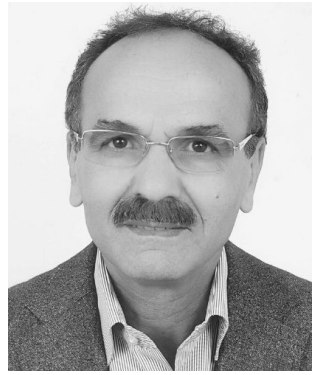

El Houssine BouyakHF graduated from Supaéro Toulouse, and he obtained his doctorate in artificial vision at the LAAS Laboratory of the CNRS in Toulouse. Avant-garde and visionary, he was convinced very early on that AI technologies would generate breakthrough innovations and a plethora of business applications thus founded LIMIARF in 1998, the first African laboratory for AI and robotics in Rabat, Morocco. He coordinated more than 40 doctoral students there, collaborated with several industrial companies and published 120 research papers in international journals and conferences. Deeply interested in the transfer of knowledge from the scientific ecosystem to the business world and feeling that more and more European and African companies are adopting data technologies, he finally founded AIOX Labs at the advent of the new era of AI. As CEO of the first spin-off company of AI laboratories in Morocco, he coordinates the development of all AI projects and products to adapt the results of his research to the needs of companies. He is also proud to create jobs for his former doctoral students that allow them to demonstrate their true values and skills.

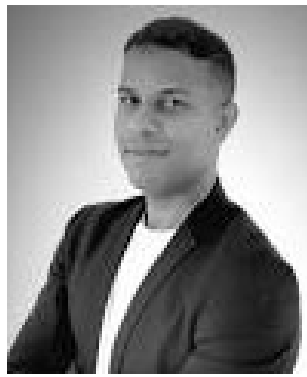

Imade Benelallam is Associate Professor, teaching data-driven courses, big data, artificial intelligence, computer science and insight data engineering at the National Institute of Statistics and Applied Economics in Rabat and other international engineering schools and universities. He is Research Director of SI2M Laboratory and Coordinator of data science engineering degree at the INSEA. He earned his Masters degrees from FS Rabat and INPT in computer science and telecom and EMI in industrial electronics. He received his Ph.D. degree in artificial intelligence and computer science from the Mohammed the Fifth

University in April 2010. His Ph.D. research focused on constraint programming and distributed constraint reasoning. His contributions aim to propose and combine new models and algorithms in constraints technology using learning for intelligent and ethical agents. He co-founded AOIX LABS, the first Artificial Intelligence startup Laboratory spin-off in Morocco. He is Active Volunteer in the IEEE association since 2004. Currently, he is appointed as IEEE Senior Member, an IBM Certified Instructor and Oracle Academy Instructor. He works in different projects within industrial partners as IT Consultant or Research Fellow; the most significant was with Thales group. He has authored several scientific publications and e-books in many conferences and journals. He served as reviewer for international journals and conferences such as Applied Soft Computing Elsevier etc. 